\title{
Anomalous origin of the left coronary artery from the pulmonary trunk: elucidation with colour Doppler flow mapping
}

\author{
A B Houston, J C S Pollock, W B Doig, J Gnanapragasam, M P G Jamieson, S Lilley, \\ E P Murtagh
}

patients with anomalous connection of the left coronary artery to the pulmonary trunk, with particular reference to its value in providing information additional to that obtained with imaging ultrasound and angiography. In addition, we report the Doppler ultrasound appearances of the coronary arteries in two infants with congestive cardiomyopathy examined after the benefits of the technique in such patients became apparent. ary artery in diastole; this latter signal localised the exact site of communication, which was not apparent on angiocardiography. Two of these patients had previously had operations for severe mitral regurgitation, the diagnosis of anomalous left coronary artery having been previously considered in one but missed despite aortic root angiography. The colour study in the fourth was largely confirmatory, operation without catheterisation being undertaken on the basis of the echocardiographic images. By contrast in two infants subsequently seen with congestive cardiomyopathy the demonstration of flow direction in the left coronary artery confirmed that it was normally connected to the aorta.

Colour Doppler flow mapping can show flow direction in the left coronary artery and from the mouth of an anomalous coronary artery into the pulmonary artery, thus simplifing the diagnosis and allowing the site of the connection of the left coronary artery to the pulmonary artery to be determined with precision.

Departments of Cardiology, Cardiac Surgery, and Child Health, Royal Hospital for Sick Children, Glasgow

A B Houston

J C S Pollock W B Doig

J Gnanapragasam M P G Jamieson $S$ Lilley

E P Murtagh

Correspondence to Dr A B Houston, Royal Hospital for Sick Children, Yorkhill, Glasgow G3 8SJ.

Accepted for publication 8 October 1989
The demonstration of the origin of an anomalous left coronary artery from the pulmonary trunk with ultrasound can be difficult and surgical correction without catheterisation has only recently been reported. ${ }^{1}$ Doppler ultrasound has the potential to show flow in or from an anomalous artery but although pulsed Doppler can show abnormal flow patterns in the pulmonary artery ${ }^{2}$ this is of limited value. Colour Doppler flow mapping will better show the site and direction of flow in or from a coronary artery in relation to the echocardiographic image, and should thus resolve diagnostic difficulties in doubtful cases.

We report on the findings with colour Doppler flow mapping in the assessment of four

\section{Patients and methods}

Imaging and Doppler studies were performed using a Vingmed CFM 700 system. A routine $M$ mode and cross sectional study was performed and when the diagnosis of anomalous connection of the left coronary artery was suspected or considered the echocardiographic investigation was directed to the demonstration of the left coronary artery. The colour Doppler was used to show the direction of flow in the left coronary artery or its main branches and to show flow from the anomalous artery into the pulmonary artery. The presence of flow signals in dilated epicardial and intraseptal collateral vessels was also sought.

The origin of the left coronary artery may be confused with the transverse sinus in the short axis view of the great arteries. ${ }^{3}$ To avoid this mistake the distal part of the artery should be sought by tilting the transducer inferiorly towards the left ventricular outflow tract. In this view more of the proximal part of the artery (probably the anterior descending) may be shown passing anteriorly. With a normal connection of the left coronary artery to the aorta the flow in this artery will be towards the transducer (red in fig 1).

\section{Results}

Four patients with anomalous connection of the left coronary artery have been diagnosed since the introduction of colour Doppler flow mapping to our unit in January 1987. One was diagnosed in infancy ( 2 months) and the others were aged 3,6, and 7 years. Cardiac catheterisation was performed in two, and surgical reimplantation of the anomalous coronary artery: in three, including one without catheterisation.

Two infants with congestive cardiomyopathy were subsequently seen and the diagnosis of an anomalous left coronary artery 


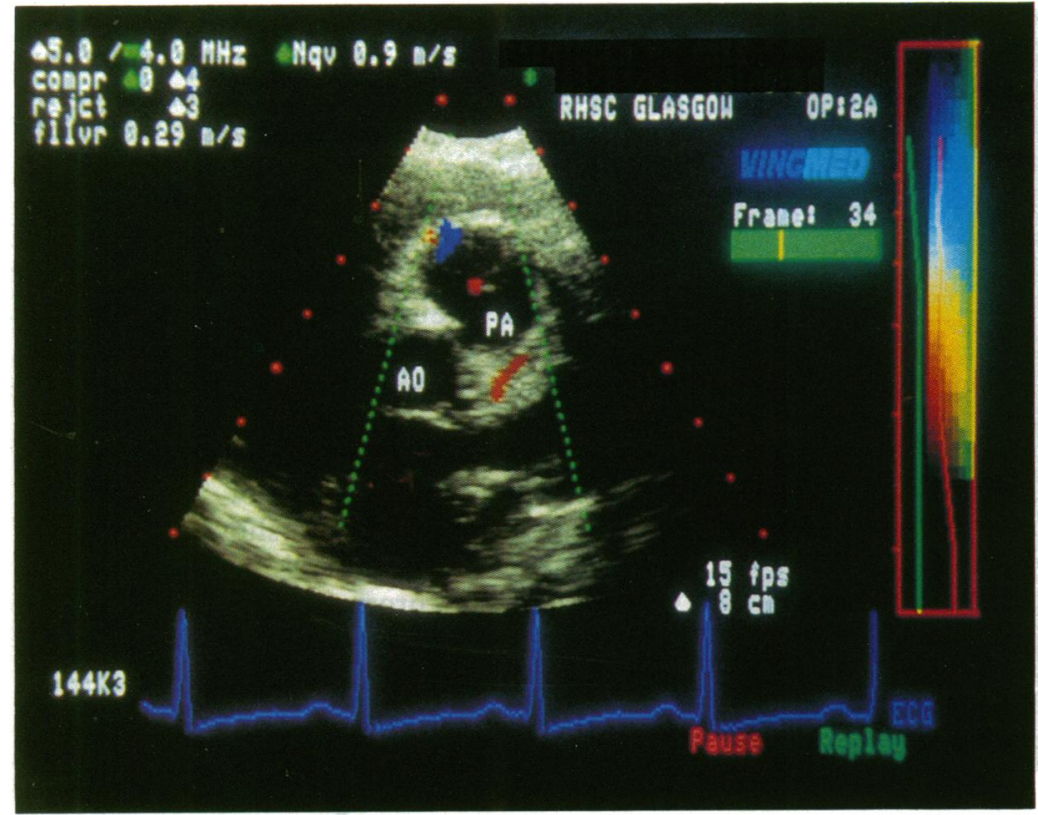

Figure 1 Colour Doppler flow mapping of modified short axis view of the great arteries in an infant with congestive cardiomyopathy showing the left main coronary artery lying behind the pulmonary artery. The ultrasound system displays flow away from the transducer as blue and towards it as red to orange. The red linear flow signal in the left coronary artery indicates flow towards the transducer. Flow is from the origin towards the bifurcation - that is, normal flow direction.

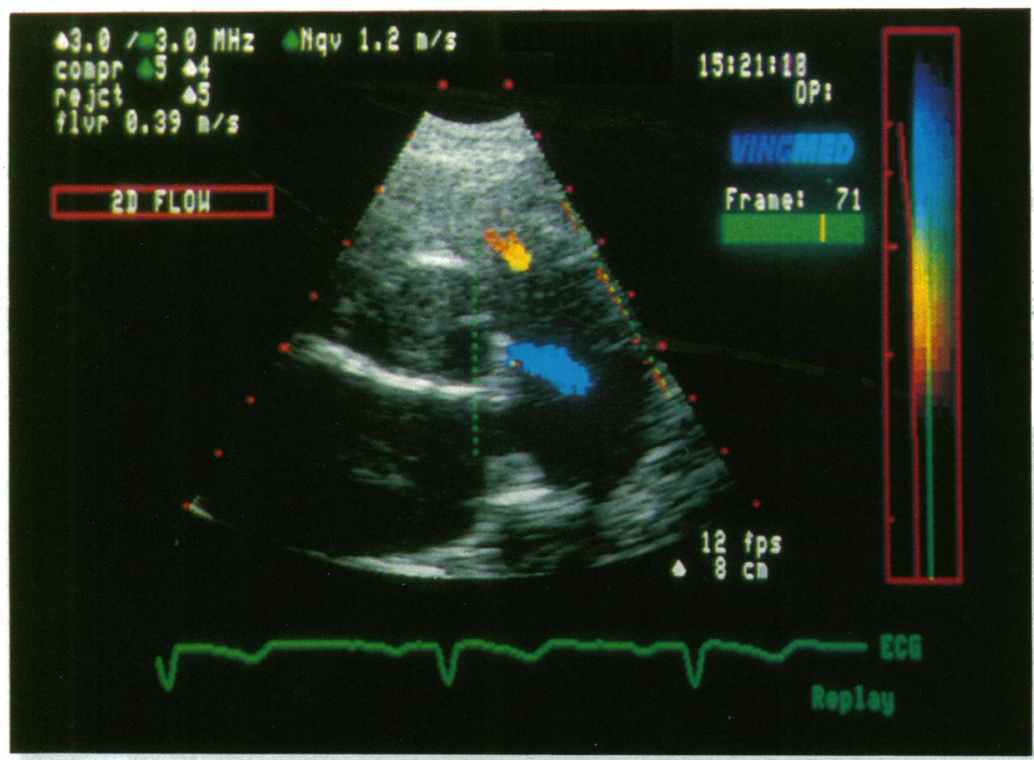

Figure 2 Colour Doppler flow mapping of short axis view of great artery origins in diastole showing flow from the anomalous coronary into the pulmonary artery (blue) just distal to the valve sinuses and insignificant pulmonary regurgitation (orange).

was considered but not confirmed. Catheterisation was undertaken in one who also had tuberous sclerosis with mitral regurgitation.

\section{ANOMALOUS LEFT CORONARY ARTERY: NO} PREVIOUS OPERATION

Two patients had clinical signs of mitral regurgitation. The first was found to have an asymptomatic murmur at 6 years of age with the electrocardiogram showing left ventricular hypertrophy but no ischaemic changes. The second presented at the age of 8 weeks with cardiac failure and ischaemic changes on the electrocardiogram. In both, echocardiography showed a dilated left ventricle with decreased contractility, affecting the posterior wall more than the septum. There were bright echoes from the papillary muscles, particularly the lateral one. An image of the aortic root initially suggested that the left coronary artery arose from the aorta, but that there was an enlarged right coronary in the infant. Spectral and colour Doppler confirmed the presence of mitral regurgitation.

Patient 1

In the first patient, the child of 6 , the diagnosis was not suspected clinically but routine colour study of the pulmonary artery showed flow from a discrete position in the medial wall just distal to the valve sinus into the centre of the vessel, most apparent in diastole (fig 2). In this view a circular echo free space was then apparent at the point of origin of the flow and adjustment of the scanning plane showed this to be an artery with flow from it into the pulmonary artery. When this artery was traced medially it was shown that although it lay in close apposition to the aorta it did not enter it, and an image could be obtained showing the artery and the structure initially thought to be the coronary artery but presumed to be the transverse sinus. ${ }^{3}$ In addition, the colour images of the septum and ventricular walls showed striking and abnormal flow signals (similar to those in fig 4 described below) resulting from flow in dilated collateral vessels.

\section{Patient 2}

In the second case, the infant, the diagnosis was suspected on the basis of the clinical signs and the initial electrocardiographic and echocardiographic studies. A colour Doppler flow mapping study of the pulmonary artery again showed flow into the main pulmonary artery similar to that in fig 2 . More detailed echocardiographic studies showed an artery arising from the pulmonary artery and bifurcating; colour Doppler flow mapping showed that the flow was from the two branches into the main one (fig 3 ) and thus from the coronary artery into the pulmonary artery. Flow in the epicardial and septal vessels was not shown, presumably because the collaterals were not developed at this young age.

In the older child catheterisation provided confirmatory images of the lesion before surgical reimplantation, which was undertaken without catheterisation in the infant.

\section{ANOMALOUS LEFT CORONARY ARTERY: PREVIOUS} MITRAL VALVE SURGERY

Two children who had undergone mitral valve repair or replacement at the ages of 6 months and 2 years were reassessed 3 and 5 years after operation.

\section{Patient 3}

One girl presented with cardiac failure and was diagnosed by angiocardiography as having endocardial fibroelastosis at the age of 4 months, but at 23 months severe mitral regurgitation with apparently improved function was diagnosed. The possibility of an anomalous coronary artery was considered but aortic root angiography was interpreted as 


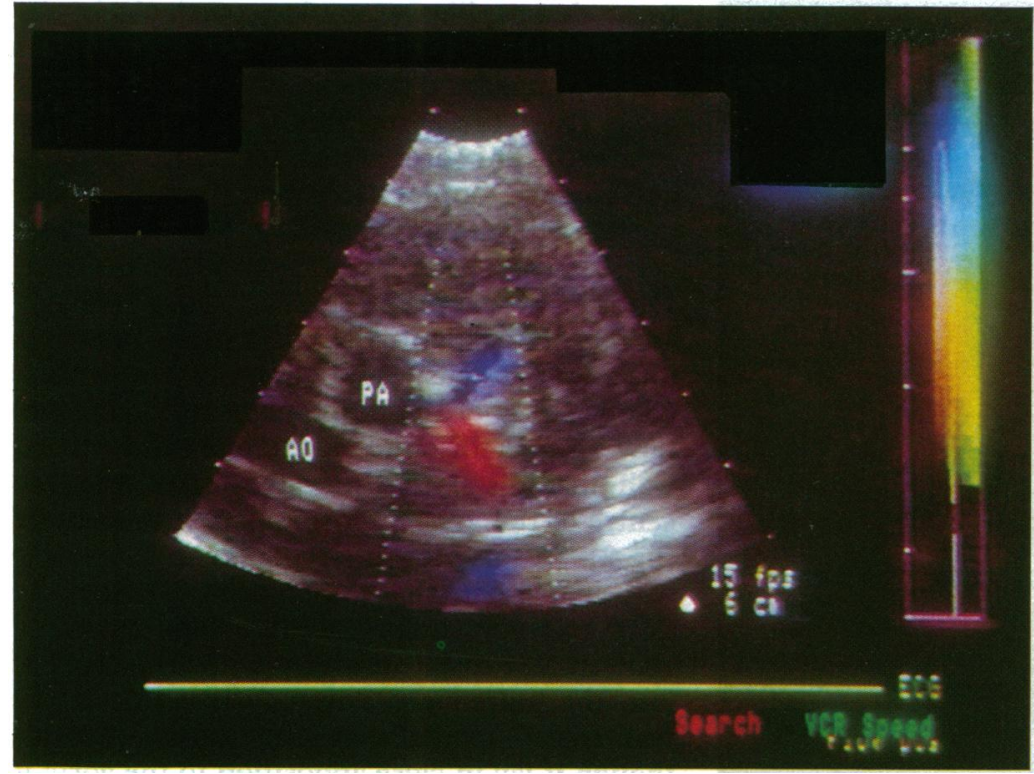

Figure 3 Colour Doppler flow mapping of still frame from video recording showing a high parasternal view of the pulmonary artery adjusted to show the anomalous vessel with flow (blue and red) from its branches towards the left main coronary artery. showing normal right and left coronaries. After mitral valve annuloplasty at 2 years 3 months her condition improved considerably. At 8 years old she was symptom free. An ultrasound study performed to assess ventricular and mitral valve function showed satisfactory ventricular function and minimal regurgitation.

The colour Doppler flow mapping study showed prominent flow signals in the septum (fig 4) and ventricular walls; these were considered to be in dilated intraseptal and epicardial vessels respectively. Study of the origins of the great arteries then showed a flow signal that was most clear in diastole passing into the right pulmonary artery just at the bifurcation.
The coronaries were then examined in detail and showed a large right coronary artery but no left coronary artery arising from the aortic root. There was also a dilated vessel with flow from it into the right pulmonary artery just distal to the bifurcation (fig 5). Subsequent right coronary angiography confirmed that there was an anomalous left coronary artery arising from the pulmonary artery, but its exact point of origin could not be ascertained. At operation the anomalous artery was found to arise from the proximal right pulmonary artery as had been shown by Doppler examination. Subsequent review of her original echocardiographic studies showed increased echogenicity of the lateral papillary muscle and a large right coronary.

\section{Patient 4}

The other patient presented at 5 months with cardiac failure and signs of mitral regurgitation. The electrocardiogram showed evidence of biventricular hypertrophy without ischaemia. Ventriculography confirmed severe mitral regurgitation and an aortic root injection was not performed. Valve replacement was undertaken and produced considerable clinical improvement. On review at 3 years he was well and the electrocardiogram showed evidence of borderline left ventricular hypertrophy but no ST changes. Colour Doppler showed dilated collateral vessels and flow into the pulmonary artery from an anomalous coronary artery.

Subsequent review of his original echocardiographic studies showed an increase in the echogenicity of the papillary muscles, particularly the lateral. He has not yet undergone surgical reimplantation.
Figure 4 Colour Doppler flow mapping of modified short axis view through the ventricles showing the ventricular septum with flow (orange) in the dilated collateral vessels in the myocardium of the septum. $L V$, left ventricle; $R V$, right ventricle.

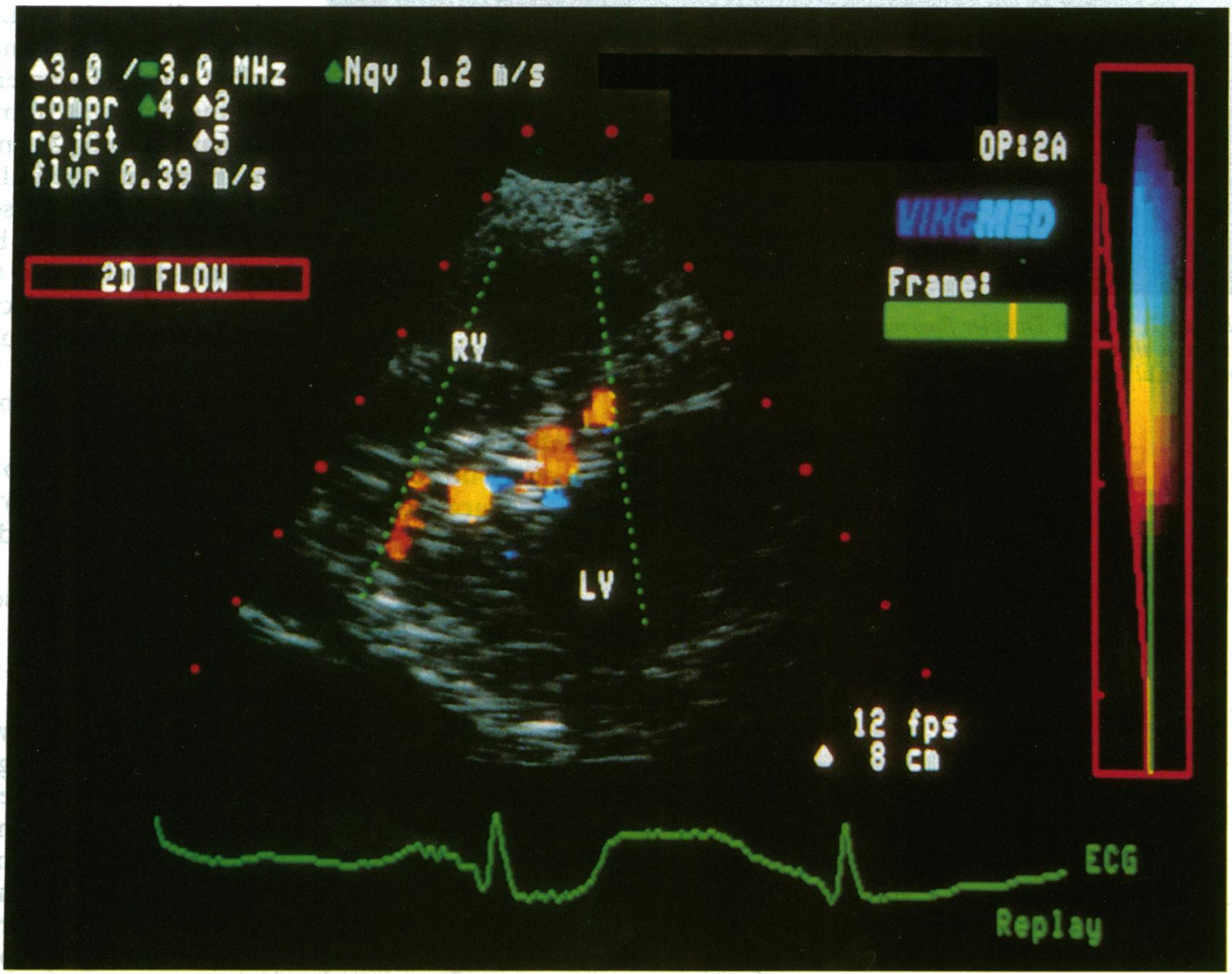


Figure 5 Colour Doppler flow mapping of short axis view of great artery origins in diastole showing flow

from the anomalous coronary into the

pulmonary artery

(orange). Note that the signal and thus the artery origin is into the right pulmonary artery ( $R P A)$ just distal to the bifurcation, different from that in figure 2. Flow in the aorta $(A O)$ is also coded in orange.

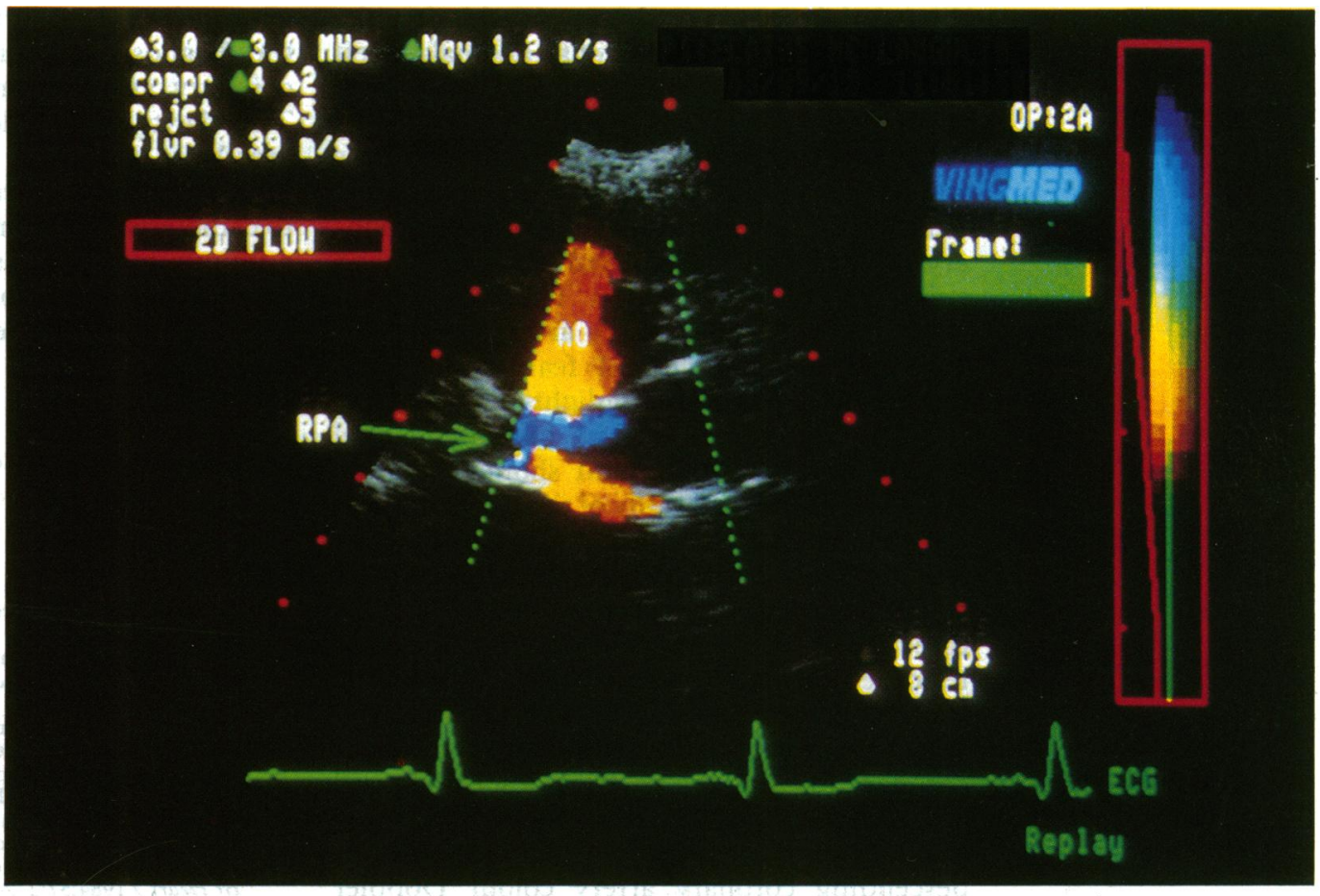

CONGESTIVE CARDIOMYOPATHY: NORMAL LEFT CORONARY ARTERY

Patient 5

An 8 month infant presented with cardiac failure, and left ventricular hypertrophy but little ischaemia on electrocardiography. Echocardiography showed a dilated and symmetrically poorly contracting left ventricle and colour Doppler showed no abnormal flow signals. Short axis views of the great arteries showed an apparently normal origin of the left coronary artery. When the scanning plane was tilted inferiorly as previously described, the anterior extension of this artery was apparent. Colour Doppler showed flow in this artery towards the transducer (fig 1) and thus from the aorta towards the distal aspect of this artery, representing a normal connection.

\section{Patient 6}

In a 2 week infant presenting with cardiac failure echocardiography showed intramyocardial tumours suggesting the diagnosis of tuberous sclerosis which was confirmed by Wood's light examination. Initial response to medical treatment was not continued and she became worse with mitral regurgitation and cardiac failure. Echocardiographic examination suggested normal origin of the coronary arteries and colour Doppler showed a normal direction of flow in the artery, thus confirming the assessment.

\section{Discussion}

Anomalous origin of a coronary artery from the pulmonary trunk is a relatively uncommon lesion. Aortic root angiography can be inconclusive $^{4}$ and the lesion may not be diagnosed without a selective injection into the right coronary artery.

It is not sufficient to find that the origin of a coronary artery from the aorta cannot be shown by echocardiography; it may be that the origin has been missed. In addition, the transverse sinus of the pericardium has been mistaken for an aortic origin of the left coronary artery. ${ }^{3}$ Very rarely there can be origin of the circumflex and right coronary arteries from the aorta with the anterior descending arising directly from the pulmonary artery. ${ }^{5}$ The presence of a dilated right coronary artery is helpful in distinguishing the lesion from dilated cardiomyopathy ${ }^{6}$ but is not diagnostic and can be found in other defects. Furthermore, although an abnormal artery may be seen it was reported some years ago that it was not always possible with imaging ultrasound to trace it into the pulmonary artery. ${ }^{7}$ Modern imaging systems make the recognition more simple but although repair on the basis of the echocardiographic image after inconclusive angiography has been reported ${ }^{8}$ we are aware of only one previous report of repair without catheterisation.

We consider that if the left coronary artery seems to arise from the aorta the scanning plane must be adjusted to show its distal course, and colour flow mapping should be used to determine whether the direction of flow is normal as in fig 1 or abnormal as in fig 3. If modern high quality imaging and colour Doppler systems are used there should be little difficulty in determining the origin and direction of flow in the left coronary artery in infants but this may be less easy in older patients. Although the flow direction will vary with the pulmonary artery pressure, this is likely to be retrograde by the time signs and symptoms of anomalous left coronary artery become apparent.

It is noteworthy that the cross sectional study in all four patients with an anomalous left coronary artery showed very bright echoes from the papillary muscles, presumably the result of ischaemic damage, which also resulted in mitral regurgitation. It thus seems that brightness of the papillary muscles and a 
dilated right coronary artery should alert the examiner to the possibility of an anomalous left coronary artery if this was not previously suspected.

In the three patients in whom the diagnosis was not suspected clinically it was only suggested from the unusual flow signal in the pulmonary artery (fig 2) or dilated myocardial and epicardial collaterals vessels. The possibility of an anomalous left coronary artery in patients with the primary clinical feature of mitral regurgitation has been highlighted by Noren $e t$ $a l .^{8}$ The appearance of flow in the collateral vessels is very striking and should alert the examiner to the possibility of the diagnosis; it is not clear at what age this becomes apparent but it is not likely in the early months before good collateral arteries have developed. The flow direction in the septal and anterior and posterior epicardial vessels was away from the transducer when it was in the subcostal and apical positions, indicating flow from the right coronary towards the anterior descending and circumflex coronary arteries. In the rare situation of anomalous origin of the anterior descending coronary artery colour Doppler should show flow into the pulmonary artery. It is to be expected that flow will be in the same direction in the septal and anterior epicardial collateral vessels but in the opposite direction (towards the transducer) in the posterior epicardial ones. This should facilitate the assessment of this condition which is otherwise very difficult to recognise.

The site of connection of the anomalous coronary to the pulmonary artery is usually just distal to the pulmonary valve ${ }^{9}$ but this is not always the case (see patient 3 ) and angiography may not show its exact position. It can then be difficult for the surgeon to locate it, and colour Doppler provides the ideal means to demonstrate clearly the site of the origin.

We consider that colour Doppler flow mapping used with good imaging is the most appropriate ultrasound means for establishing the diagnosis of anomalous origin of the left coronary artery from the pulmonary artery. The clarity of this demonstration, by adding greatly to the other echocardiographic findings, will allow surgical repair without recourse to catheterisation in many patients. It provides a unique means to ascertain the exact site of origin of the anomalous artery and thus assists the surgeon in the technical approach to the procedure.

JG is in receipt of a grant from the Equipment Evaluation Committee of the Scottish Home and Health Department.

1 Sreeram N, Hunter $S, W$ ren C. Acute myocardial infarction in infancy: unmasking of anomalous origin of the left coronary artery from the pulmonary artery by ligation of coronary artery from the pulmonary artery

2 King DH, Danfard DA, Huhta JC, Gutgesell HP. Noninvasive detection of anomalous origin of the left main coronary artery from the pulmonary trunk by pulsed Doppler echocardiography. Am J Cardiol 1985;55:608-9.

3 Robinson PJ, Sullivan ID, Kumpeng V, Anderson RH, Macartney FJ. Anomalous origin of the left coronary artery from the pulmonary trunk: potential for false negative diagnosis with cross sectional echocardiography. Br Heart J 1984;52:272-7.

4 Jureidini SB, Nouri S, Pennington DG. Anomalous origin of the left coronary artery from the pulmonary trunk: repair after diagnostic cross sectional echocardiography. $\mathrm{Br}$ Heart $J$ 1987;58:173-5.

5 El Habbal MM, de Laval M, Somerville J. Anomalous origin of the left anterior descending coronary artery from the pulmonary trunk: recognition in life and successful surgical treatment. Br Heart J 1988;60:90-2.

6 Koike K, Musewe NN, Smallhorn JF, Freedom RM Distinguishing between anomalous origin of the left coronary artery from the pulmonary trunk and dilated cardiomyopathy: role of echocardiographic measurement cardiomyopathy: role of echocardiographic measurement
of the right artery diameter. Br Heart $J$ 1989;61:192-7.

7 Caldwell RL, Hurwitz RA, Girod DA, Weyman AE Feigenbaum $H$. Two-dimensional echocardiographic differentiation of anomalous left coronary artery from congestive cardiomyopathy. Am Heart J 1983;106:710-6.

8 Noren GR, Raghib G, Moller JH, Amplatz K, Adams P, Edwards JE. Anomalous origin of the left coronary artery from the pulmonary trunk with special reference to the occurrence of mitral insufficiency. Circulation 1964;30:171-9.

9 Wesselhoeft H, Fawcett JS, Johnson AL. Anomalous origin of the left coronary artery from the pulmonary trunk. Its clinical spectrum, pathology, and pathophysiology, based on a review of 140 cases with seven further cases. 